\title{
ÍNDICES ANTROPOMÉTRICOS PARA LA EVALUACIÓN DE LA EMBARAZADA Y EL RECIÉN NACIDO: CÁLCULO MEDIANTE TABLAS BIDIMENSIONALES
}

\author{
Rudecindo Lagos S. 1,2,3, Ximena Ossa G. ${ }^{2,3}$, Luis Bustos M. ${ }^{\text {a,2,3, }}$ \\ Juan Orellana C. ${ }^{a, 2,3}$ \\ a Bioestadísticos \\ 1 Maternidad del Hospital Regional de Temuco. ${ }^{2}$ Capacitación Investigación y Gestión para la Salud Basada en Eviden- \\ cia (CIGES), Facultad de Medicina, Universidad de la Frontera. 3 Departamento de Salud Pública, Facultad de Medicina, \\ Universidad de la Frontera, Temuco, Chile.
}

\section{RESUMEN}

Antecedentes: La valoración nutricional de la madre y el recién nacido es un criterio ampliamente usado con fines pronósticos y de manejo clínico. En el ámbito obstétrico materno y neonatal se utiliza índice de masa corporal e índice ponderal neonatal respectivamente. Objetivo: Construir tablas bidimensionales que en la clínica práctica faciliten la obtención de estos índices. Método: Los índices antropométricos que relacionan el peso y la talla, fueron calculados mediante sus fórmulas respectivas: peso dividido por talla al cuadrado o al cubo según se trate de índice de masa corporal o índice ponderal neonatal. Para la categorización posterior del estado nutricional tanto materno como neonatal, los índices obtenidos han de ser valorados con gráficas en función de la edad gestacional. Recomendamos para ello los estándares de referencia nacional. Resultados: Se conformaron dos tablas bidimensionales para el cálculo de índices, las cuales fueron acotadas de acuerdo a valores antropométricos promedios de nuestra población. Conclusión: Estas tablas facilitarán al clínico la obtención de índices antropométricos y el diagnóstico de la condición nutricional de la embarazada y recién nacido.

\section{PALABRAS CLAVES: Diagnóstico nutricional, índices antropométricos}

\section{SUMMARY}

Background: Nutritional assessment is a widely used criterion for prognostic purposes and clinical management. In obstetrics, maternal and neonatal body mass index and neonatal ponderal index, respectively, are used. Objective: To construct bidimensional tables to facilitate indexes obtaining process in clinical practice, moreover, graphs also are recommend for nutritional categorize using this indexes. Methods: The anthropometric indexes that associate weight with height were calculated by their respectively formulas: weight divided by height squared or cubed, depending if body mass index or neonatal ponderal index is needed. For the subsequently categorization of both maternal and neonatal nutritional status, these indexes have to be judged in terms of gestational age graphics. For this process, we recommend national standard references. Results: Two bidimensional tables were built to calculate the indexes and were delimited according to anthropometric averages of our population. Conclusion: These tables will facilitate the obtaining of indexes by the clinicians and, at the same time, the nutritional status diagnosis of pregnant women and their newborn.

KEY WORDS: Nutritional assessment, anthropometric indexes 


\section{INTRODUCCIÓN}

En medicina materno fetal la valoración nutricional es un criterio ampliamente utilizado con fines pronósticos y de manejo clínico (1-4), la desnutrición materna tradicionalmente ha sido considerada un factor de riesgo para el normal desarrollo del feto. El sobrepeso y la obesidad materna representan mayor riesgo perinatal, aumentando el riesgo en términos de macrosomia y partos distócicos. Además, la persistencia de sobrepeso materno en el posparto aumenta el riesgo materno de diabetes, hipertensión y otras enfermedades crónicas (5-8).

Para la valoración nutricional materna se utilizan índices de la relación peso/talla. En Chile, el primer índice utilizado fue el porcentaje de peso/talla (PPT) (9), luego el índice de masa corporal (IMC=peso/ta$\mathrm{Ila}^{2}$ ), recomendado por el Instituto de Medicina de la Academia Nacional de Ciencias (10), para así estandarizar la medición en el adulto. Posteriormente, el índice peso/talla fue reconvertido por los mismos autores a índice de masa corporal (11).

El Ministerio de Salud de Chile (MINSAL), actualmente recomienda para la valoración del estado nutricional de las gestantes chilenas, la utilización del IMC, y para la categorización de las mismas, recomienda el estándar nutricional propuesto por Atalah y cols $(12)$. Ambos estándares $(9,12)$ clasifican a la embarazada en cuatro categorías: enflaquecida, normal, sobrepeso u obesa.

El cálculo del IMC de la gestante en la práctica clínica diaria requiere de un ejercicio matemático o de un procedimiento a través de nomograma, el cual requiere cierto tiempo y dedicación para evitar sesgos de clasificación.

En el campo fetal, la primera aplicación de la ecografía al reconocimiento de la proporcionalidad corporal anormal, a objeto de pesquisar desnutrición in útero, fue comunicada en 1977 por Campbell y Thoms (13), utilizando la relación perímetro cefálico/perímetro abdominal, índice que varía en función de la edad gestacional. En 1983, Hadlock y cols (14) proponen una evaluación de la relación entre el perímetro abdominal (como indicador de peso fetal) y la longitud del fémur (como indicador de la longitud fetal). Dado que la relación fémur/ abdomen permanece constante desde las 20 semanas, este indicador es útil para la búsqueda de crecimientos asimétricos en aquellos embarazos de tercer trimestre con edad gestacional incierta. Yagel y cols (15), evaluaron el índice ponderal in útero (IPIU), que relaciona el peso fetal estimado (PFE) y la longitud del fémur al cubo (IPIU=PFE/ $\mathrm{LF}^{3}$ ); observaron que el bienestar de los fetos y recién nacido estaban comprometidos en casos de
RCIU con IPIU alterado, situación similar a lo observado cuando la relación fémur/abdomen estaba alterada.

Por otro lado para la valoración neonatal del crecimiento intrauterino, tradicionalmente se ha utilizado el criterio de Battaglia y Lubchenco $(2,16-$ 21), relacionando el peso del recién nacido $(R N)$ en función de la edad gestacional. Este procedimiento permite clasificar al RN en pequeño (PEG), adecuado (AEG) o grande (GEG) para la edad gestacional, según se encuentre bajo el percentil 10, entre 10 y 90 o sobre percentil 90 respectivamente. Sin embargo, una mejor evaluación del estado nutricional del RN se logra aplicando el índice ponderal neonatal $\left[\left(\mathrm{IPN}=\right.\right.$ peso/talla $\left.{ }^{3}{ }^{\star} 100\right]$ propuesto por Rohrer en 1960 (22-25). El uso de este indicador permite valorar de mejor forma el estado nutricional neonatal (eutrófico, enflaquecido $u$ obeso), siendo un complemento importante a la categorización tradicional del recién nacido; peso/edad gestacional (AEG, PEG o GEG).

El cálculo del IPN en la práctica clínica también requiere de un ejercicio matemático personal del profesional a cargo, lo que implica tiempo y dedicación para evitar sesgos de clasificación, en este caso, no se dispone de nomograma.

El objetivo de este trabajo es facilitar la obtención de índices maternos y neonatales a través de tablas bidimensionales, en unidades del sistema métrico decimal y, por consecuencia, la categorización nutricional materna y neonatal, independiente de la gráfica de referencia utilizada.

\section{MATERIAL Y MÉTODO}

Se construyeron 2 tablas bidimensionales, una para el cálculo del índice de masa corporal materna y otra para el cálculo del índice ponderal neonatal. Tabla de cálculo del índice de masa corporal materno: este instrumento relaciona el peso materno en kilogramos dividido por su talla en metros elevada al cuadrado, obteniendo así el índice de masa corporal materno $\left(\mathrm{IMC}=\right.$ peso/talla $\left.{ }^{2}\right)$.

Tabla de cálculo del índice ponderal neonatal: este instrumento relaciona el peso del recién nacido en gramos dividido por la talla en centímetros elevada al cubo, obteniéndose el índice ponderal neonatal $\left[(I P N=\text { peso/talla })^{*} 100\right]$. EI IPN se multiplica por 100 para obtener unidades de uso habitual, un entero y dos decimales.

Ambas tablas se construyeron mediante función matemática en Excel 2007 de Microsoft Office, combinando las medidas antropométricas maternas y neonatales de peso y talla.

Para su aplicación clínica, estos índices deben 
ser llevados a gráficas específicas para categorizar el estado nutricional en función de la edad gestacional:

a) Para la categorización del estado nutricional materno se utilizaron los estándares de referencia propuesto por Atalah y cols (12) (Figura 1). Con valores de referencia para la semana gestacional 10 a la 40 . b) Para la categorización del estado nutricional neonatal se utilizaron los estándares de referencia propuesto por Milad y cols (21) (Figura 2). Con valores de referencia para la semana 24 a la 42.

Las tablas bidimensionales fueron acotadas de acuerdo a valores antropométricos promedios de nuestra población (16-21), utilizando valores máximos y mínimos de acuerdo a los referentes nacionales maternos y neonatales antes señalados.

\section{RESULTADOS}

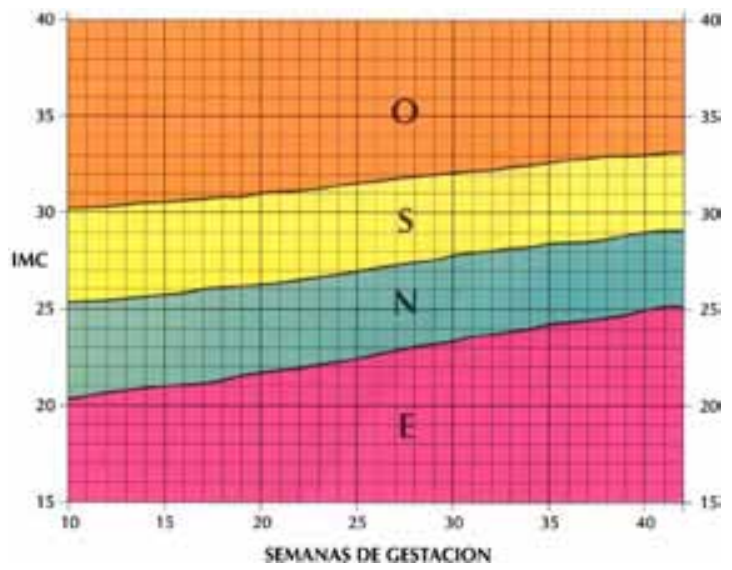

Figura 1. Categorización del índice de masa corporal materna (IMC) en función de la edad gestacional (12).

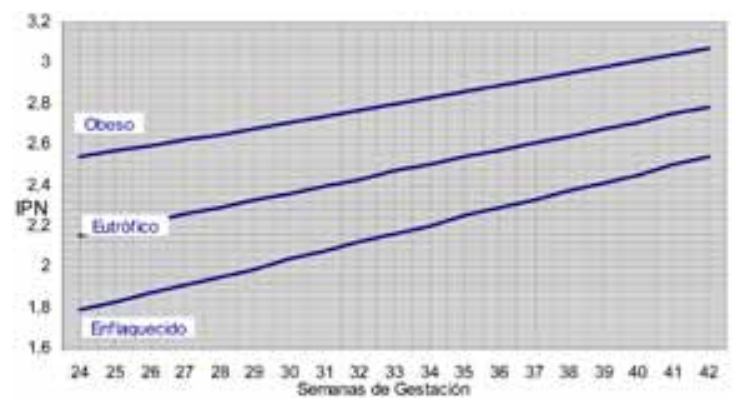

Figura 2. Categorización del índice ponderal neonatal (IPN) en función de la edad gestacional (21).
En las Tablas $1 \mathrm{~A}$ y $1 \mathrm{~B}$ se presentan las tablas bidimensionales para el cálculo índice de masa corporal materno, donde en el eje vertical se ubica la talla centímetros y en la horizontal, el peso en kilos, obteniendo en la conjunción de los valores el índice de masa corporal respectivo.

Para la categorización nutricional de la embarazada, el índice de masa corporal calculado anteriormente se ubica según edad gestacional en gráfica propuesta por Atalah y cols (12), obteniendo según sea el caso, el diagnóstico de enflaquecida, normal, sobrepeso u obesa.

En las Tablas $2 \mathrm{~A}$ y $2 \mathrm{~B}$ se presentan las tablas bidimensionales para el cálculo del índice ponderal neonatal, donde en eje vertical se ubica la talla del recién nacido en centímetros y en la horizontal, el peso en gramos, obteniendo en la conjunción de los valores el índice ponderal neonatal respectivo.

Para la categorización nutricional del recién nacido, el índice ponderal neonatal calculado anteriormente se ubica según edad gestacional en la gráfica propuesta por Milad y cols (21), obteniendo según sea el caso el diagnóstico de enflaquecido, eutrófico u obeso.

\section{DISCUSIÓN}

La confección de las tablas bidimensionales facilitará la obtención de índices antropométricos maternos y neonatales necesarios para el diagnóstico nutricional respectivo, favoreciendo así su utilización clínica.

La simplificación del ejercicio matemático a un procedimiento mecánico para el cálculo de los índices antropométricos, especialmente en lugares donde no se cuenta con el recurso tecnológico adecuado, reduce el riesgo de sesgos en la clasificación nutricional de la embarazada y su recién nacido, y reduce el tiempo de operación optimizando el tiempo/desempeño del profesional, de allí, el aporte de ofrecer estas tablas bidimensionales construidas con fórmulas estandarizadas, utilizando metros o centímetros para la talla y kilos o gramos para el peso, según se trate de adultos o recién nacidos facilitando al clínico la obtención de los índices antropométricos.

\section{CONCLUSIÓN}

Las tablas bidimensionales presentadas, facilitarán al clínico la obtención de índices antropométricos y el diagnóstico de la condición nutricional de la embarazada y su recién nacido, especialmente donde no se dispone de métodos electrónicos para su determinación. 


\section{Tabla I A}

\section{Tabla de cálculo del Índice de masa corporal materna}

\begin{tabular}{|c|c|c|c|c|c|c|c|c|c|c|c|c|c|c|c|c|c|c|c|c|c|c|c|c|c|c|c|c|c|c|c|c|}
\hline \multirow{2}{*}{$\begin{array}{l}\text { Talla } \\
\text { Mtr. }\end{array}$} & \multicolumn{31}{|c|}{ Peso Materno en kilos. } & \multirow{2}{*}{$\begin{array}{r}\text { Talla } \\
\text { Mtr. }\end{array}$} \\
\hline & 40 & 42 & 44 & 46 & 48 & 50 & 52 & 54 & 56 & 58 & 60 & 62 & 64 & 66 & 68 & 70 & 72 & 74 & 76 & 78 & 80 & 82 & 84 & 86 & 88 & 90 & 92 & 94 & 96 & 98 & 100 & \\
\hline 1,4 & 4 & 21,4 & 22,4 & 23,5 & 24,5 & 25,5 & 26,5 & 27,6 & 28,6 & 29,6 & 30,6 & 31,6 & 32,7 & 33,7 & 34,7 & 35,7 & 36,7 & 37,8 & 38,8 & 39,8 & 40,8 & 41,8 & 42,9 & 43,9 & 44,9 & 45,9 & 46,9 & 48,0 & 49,0 & 50,0 & 51, & 4 \\
\hline 1,41 & 0,1 & 21,1 & 22,1 & 23,1 & 24,1 & 25,1 & 26,2 & 27,2 & 28,2 & 29,2 & 30,2 & 31,2 & 32,2 & 33,2 & 34,2 & 35,2 & 36,2 & 37,2 & 38,2 & 39,2 & 40,2 & 41,2 & 42,3 & 43,3 & 44,3 & 45,3 & 46,3 & 47,3 & 48,3 & 49,3 & 50,3 & 1,41 \\
\hline 1,42 & 8 & 20,8 & 21,8 & 22,8 & 23,8 & 24,8 & 25,8 & 26,8 & 27,8 & 28,8 & 29,8 & 30,7 & 31,7 & 32,7 & 33,7 & 34,7 & 35,7 & 36,7 & 37,7 & 38,7 & 39,7 & 40,7 & 41,7 & 42,7 & 43,6 & 44,6 & 45,6 & 46,6 & 47,6 & 48,6 & 49,6 & 1,42 \\
\hline 43 & 9,6 & 20,5 & 21,5 & 22,5 & 23,5 & 24,5 & 25,4 & 26,4 & 27,4 & 28,4 & 29,3 & 30,3 & 31,3 & 32,3 & 33,3 & 34,2 & 35,2 & 36,2 & 37,2 & 38,1 & 39,1 & 40,1 & 41,1 & 42,1 & 43,0 & 44,0 & 45,0 & 46,0 & 46,9 & 47,9 & 48,9 & 1,43 \\
\hline 44 & 3 & 20,3 & 21,2 & 22,2 & 23,1 & 24,1 & 25,1 & 26,0 & 27,0 & 28,0 & 28,9 & 29,9 & 30,9 & 31,8 & 32,8 & 33,8 & 34,7 & 35,7 & 36,7 & 37,6 & 38,6 & 39,5 & 40,5 & 41,5 & 42,4 & 43,4 & 44,4 & 45,3 & 46,3 & 47,3 & 48,2 & ,44 \\
\hline 1,45 & 0 & 20,0 & 20,9 & 21,9 & 22,8 & 23,8 & 24,7 & 25,7 & 26,6 & 27,6 & 28,5 & 29,5 & 30,4 & 31,4 & 32,3 & 33,3 & 34,2 & 35,2 & 36,1 & 37,1 & 38,0 & 39,0 & 40,0 & 40,9 & 41,9 & 42,8 & 43,8 & 44,7 & 45,7 & 46,6 & 47,6 & 1,45 \\
\hline 1,46 & B,8 & 19,7 & 20,6 & 21,6 & 22,5 & 23,5 & 24,4 & 25,3 & 26,3 & 27,2 & 28,1 & 29,1 & 30,0 & 0 & 31,9 & 32,8 & 33,8 & 34,7 & 35,7 & 36,6 & 37,5 & 38,5 & 39,4 & 40,3 & 41,3 & 42,2 & 43,2 & 44,1 & 45,0 & 46,0 & 46, & 1,46 \\
\hline 1,47 &, 5 & 19,4 & 20,4 & 21,3 & 22,2 & 23,1 & 24,1 & 25,0 & 25,9 & 26,8 & 27,8 & 28,7 & 29,6 & 30,5 & 31,5 & 32,4 & 33,3 & 34,2 & 35,2 & 36,1 & 37,0 & 37,9 & 38,9 & 39,8 & 40,7 & 41,6 & 42,6 & 43,5 & 44,4 & 45,4 & 46,3 & 1,47 \\
\hline 48 & 3 & 19,2 & 20,1 & 21,0 & 21,9 & 22,8 & 23,7 & 24,7 & 25,6 & 26,5 & 27,4 & 28,3 & 29,2 & 30,1 & 1,0 & 32,0 & 32,9 & 33,8 & 34,7 & 35,6 & 36,5 & 37,4 & 38,3 & 39,3 & 40,2 & 41,1 & 42,0 & 42,9 & 43,8 & 44,7 & 45,7 & 1,48 \\
\hline 49 & 0 & 18,9 & 19,8 & 20,7 & 21,6 & 22,5 & 23,4 & 24,3 ] & 25,2 & 1 & 27,0 & 27,9 & 28,8 & 29,7 & 30,6 & 31,5 & 32,4 & 33,3 & 34,2 & 35,1 & 36,0 & 36,9 & 37,8 & 38,7 & 39,6 & 40,5 & 41,4 & 42,3 & 43,2 & 44,1 & $45, \mathrm{C}$ & 1,49 \\
\hline 1 , & 7,8 & 18,7 & 19,6 & 20,4 & 21,3 & 22,2 & 23,1 & 24,0 & 24,9 & 25,8 & 26,7 & 27,6 & 28,4 & 29,3 & 30,2 & 31,1 & 32,0 & 32,9 & 33,8 & 34,7 & 35,6 & 36,4 & 37,3 & 38,2 & 39,1 & 40,0 & 40,9 & 41,8 & 42,7 & 43,6 & 44, & ,5 \\
\hline 51 & .5 & 18,4 & 19,3 & 20,2 & 2 & 21,9 & 22,8 & 3,7 & 24,6 & 25,4 & 26,3 & 27,2 & 28,1 & 28,9 & 29,8 & 30,7 & 31,6 & 32,5 & 33,3 & 34,2 & 35,1 & 36,0 & 36,8 & 37,7 & 38,6 & 39,5 & 3 & 41,2 & 42,1 & 43,0 & 43, & ,51 \\
\hline 1,52 & 3 & 18,2 & 19,0 & 19,9 & 20,8 & 21,6 & 22,5 & 23,4 & 24,2 & \begin{tabular}{|l}
25,1 \\
\end{tabular} & 26,0 & 26,8 & 27,7 & 28,6 & 29,4 & 30,3 & 31,2 & 32,0 & 32,9 & 33,8 & 34,6 & 35,5 & 36,4 & 37,2 & 38,1 & 39,0 & 39,8 & 40,7 & 41,6 & 42,4 & 43,3 & 1,5 \\
\hline 1,53 & , & 17,9 & 18,8 & 19,7 & 20,5 & 21,4 & 22,2 & 23,1 & 23,9 & 24,8 & 25,6 & 26,5 & 27,3 & 28,2 & 29,0 & 29,9 & 30,8 & 31,6 & 32,5 & 33,3 & 34,2 & 35,0 & 35,9 & 36,7 & 37,6 & 38,4 & 39,3 & 40,2 & 41,0 & 41,9 & 42,7 & 1,53 \\
\hline 1,54 & ,9 & 17,7 & 18,6 & 19,4 & 20,2 & 21,1 & 21,9 & 22,8 & 23,6 & 24,5 & 2 & 26,1 & 27,0 & 27,8 & $20, r$ & 2 & 3 & 31,2 & 32,0 & & 7 & 6 & 35,4 & 36,3 & 37,1 & 37,9 & 8 & 6 &, 5 & 41,3 & 42 & ,54 \\
\hline 1,55 & $; 6$ & 17,5 & 18,3 & 19,1 & 20,0 & 20,8 & 21,6 & 22,5 & 23,3 & 24,1 & 25,0 & 25,8 & 26,6 & 27,5 & 28,3 & 29,1 & 30,0 & 30,8 & 31,6 & 32,5 & 33,3 & 34,1 & 35,0 & 35,8 & 36,6 & 37,5 & 38,3 & 39,1 & 40,0 & 40,8 & 41,6 & 1,55 \\
\hline 1,56 & ,4 & 17,3 & 18,1 & 18,9 & 19,7 & 20,5 & 21,4 & 22,2 & 23,0 & 23,8 & 24,7 & 25,5 & 26,3 & 27,1 & 27,9 & 28,8 & 29,6 & 30,4 & 31,2 & 32,1 & 32,9 & 33,7 & 34,5 & 35,3 & 36,2 & 37,0 & 37,8 & 38,6 & 39,4 & 40,3 & 41,1 & ,56 \\
\hline 1,57 & ,2 & 17,0 & 17,9 & 18,7 & 19,5 & 20,3 & 21,1 & 21,9 & 22,7 & 23,5 & 24,3 & 25,2 & 26,0 & 26,8 & 27,6 & 28,4 & 29,2 & 30,0 & 30,8 & 31,6 & 32,5 & 33,3 & 34,1 & 34,9 & 35,7 & 36,5 & 37,3 & 38,1 & 38,9 & 39,8 & 40,6 & I I \\
\hline 1,58 & 5,0 & 16,8 & 17,6 & 18,4 & 19,2 & 20,0 & 20,8 & 21,6 & 22,4 & 23,2 & 24,0 & 24,8 & 25,6 & 26,4 & 27,2 & 28,0 & 28,8 & 29,6 & 30,4 & 31,2 & 32,0 & 32,8 & 33,6 & 34,4 & 35,3 & 36,1 & 36,9 & 37,7 & 38,5 & 39,3 & 40,1 & 1,58 \\
\hline 1,59 & 15,8 & 16,6 & 17,4 & 18,2 & 19,0 & 19,8 & 20,6 & 21,4 & 22,2 & 22,9 & 23,7 & 24,5 & 25,3 & 26,1 & 26,9 & 27,7 & 28,5 & 29,3 & 30,1 & 30,9 & 31,6 & 32,4 & 33,2 & 34,0 & 34,8 & 35,6 & 36,4 & 37,2 & 38,0 & 38,8 & 39,6 & 1,59 \\
\hline 1,6 & 15,6 & 16,4 & 17,2 & 18,0 & 18,8 & 19,5 & 20,3 & 21,1 & 21,9 & 22,7 & 23,4 & 24,2 & 25,0 & 25,8 & 26,6 & 27,3 & 28,1 & 28,9 & 29,7 & 30,5 & 31,3 & 32,0 & 32,8 & 33,6 & 34,4 & 35,2 & 35,9 & 36,7 & 37,5 & 38,3 & 39,1 & 1,6 \\
\hline & & ENF & A & II & & & & & $\mathrm{N}$ & SMAL & & & & SOBF & $2 \mathrm{E}$ & ESO & & & & & & & & & & & & & & & & \\
\hline
\end{tabular}

\section{Tabla I B}

Tabla de cálculo del Índice de masa corporal materna

\begin{tabular}{|c|c|c|c|c|c|c|c|c|c|c|c|c|c|c|c|c|c|c|c|c|c|c|c|c|c|c|c|c|c|c|c|c|}
\hline \multirow{2}{*}{$\begin{array}{l}\text { Talla } \\
\text { Mtr. }\end{array}$} & \multicolumn{31}{|c|}{ Peso Materno en kilos. } & \multirow{2}{*}{$\begin{array}{l}\text { Talla } \\
\text { Mtr. }\end{array}$} \\
\hline & 40 & 2 & 14 & 46 & 48 & 50 & 52 & 54 & 56 & 58 & 60 & 62 & 64 & 66 & 68 & 70 & 72 & 74 & 76 & 78 & 80 & 82 & 84 & 86 & 88 & 0 & 92 & 94 & 96 & 98 & 100 & \\
\hline 1,61 & 4 & 16,2 & 17,0 & 17,7 & 18,5 & 19,3 & 20,1 & 20,8 & 21,6 & 22,4 & 23,1 & 23,9 & 24,7 & 25,5 & 26,2 & 27,0 & 27,8 & 28,5 & 29,3 & 30 & 1,9 & 1,6 & 32,4 & 33,2 & 33,9 & 34,7 & 35,5 & 36,3 & 37,0 & 37,8 & 3,6 & 1,61 \\
\hline 62 & & & & & 18,3 & 19,1 & 19,8 & 6 & 21,3 & 22,1 & 22,9 & 23,6 & 24,4 & 1 & 25,9 & 26,7 & 27,4 & 28,2 & 29,0 & 29,7 & 30,5 & 31,2 & 32,0 & 32,8 & 33,5 & 34,3 & 35,1 & 35,8 & 36,6 & 37,3 & 3 & ,62 \\
\hline 63 & 1 & 15,8 & 16,6 & 17,3 & 18,1 & 18,8 & 19,6 & 20,3 & 21,1 & 21,8 & 22,6 & 23,3 & 24,1 & 24,8 & 25,6 & 26,3 & 27,1 & 27,9 & 28,6 & 29,4 & 30,1 & 30,9 & 31,6 & 32,4 & 33,1 & 33,9 & 34,6 & 35,4 & 436,1 & 36,9 & $\begin{array}{l}97,6 \\
\end{array}$ & I,63 \\
\hline 1,04 & 9 & 15,6 & 16,4 & 17,1 & 17,8 & 18,6 & 19,3 & 20,1 & 20,8 & 21,6 & 22,3 & 23,1 & 23,8 & 24,5 & 25,3 & 26,0 & 26,8 & 27,5 & 28,3 & 29,0 & 29,7 & 30,5 & 31,2 & 32,0 & 32,7 & 33,5 & 34,2 & 34,9 & 9 35,7 & 36,4 & $4 \quad 37,2$ & ,64 \\
\hline 1,6 & 7 & 1 & 1 & 16 & 17 & 18,4 & 19,1 & 19,8 & 2 & 2 & 0 & 22,8 & 23,5 & 24,2 L & 25,0 & 7 & 26,4 & 27,2 & 27,9 & 28,7 & 29,4 & 1 & 9 & 31,6 & 32,3 & 33,1 & 8 &, 5 & 535,3 & 36,0 & D & 1,65 \\
\hline 1,66 & 5 & 15,2 & 16,0 & 16,7 & 17,4 & 18,1 & 18,9 & 19,6 & 20,3 & 21,0 & 21,8 & 22,5 & 23,2 & 24,0 & 24,7 & 25,4 & 26,1 & 26,9 & 27,6 & 28,3 & 29,0 & 29,8 & 30,5 & 31,2 & 31,9 & 32,7 & 33,4 & 34,1 & 134,8 & 35,6 & 36,3 & ,66 \\
\hline 1,67 & 3 & 15,1 & 15,8 & 16,5 & 17,2 & 17,9 & 18,6 & 19,4 & 20,1 & 20,8 & 21,5 & 22,2 & 22,9 & 23,7 & 24,4 & 25,1 & 25,8 & 26,5 & 27,3 & 28,0 & 28,7 & 29,4 & 30,1 & 30,8 & 31,6 & 32,3 & 33,0 & 33,7 & $7 \quad 34,4$ & 35,1 & 35,9 & 1,67 \\
\hline 1,68 & & & & & 17 & 1 & 18 & 19,1 & 19,8 & 2 & 21 & 22 & 22,7 & 23 & 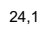 & 24,8 & 25,5 & 26,2 & 26,9 & 27,6 & 28,3 & 29,1 & 29,8 & 30,5 & 31,2 & 31,9 & 6 & 3,3 & 34,0 & 34,7 & 7 & ,68 \\
\hline 69 & 0 & 14,7 & 15,4 & 16,1 & 16,8 & 17,5 & 18,2 & 18,9 & 19,6 & 20,3 & 21,0 & 21,7 & 22,4 & 23,1 & 23,8 & 24,5 & 25,2 & 25,9 & 26,6 & 27,3 & 28,0 & 28,7 & 29,4 & 30,1 & 30,8 & 31,5 & 32,2 & 32,9 & 933,6 & 34,3 & $\begin{array}{ll}3 & 35,0\end{array}$ & 1,69 \\
\hline 1,7 & 38 & 1 & 1 & 1 & 1 & 3 & 0 & 7 & 4 & 20,1 & 8 & 2 & 22,1 & 8 & 5 & 2 & 24,9 & 25,6 & 26,3 & 27,0 & 27,7 & 28,4 & 29,1 & 29,8 & 30,4 & 31,1 & 31,8 & 32,5 & 53,2 & 2 33,9 & 93 & ,7 \\
\hline 1,71 & & 1 & 1 & 1 & 16,4 & 1 & 17,8 & 3,5 & 9,2 & 19,8 & 20,5 & 21,2 & 21,9 & 22,6 & 23,3 & 23,9 & 24,6 & 25,3 & 26,0 & 26,7 & 27,4 & 28,0 & 28,7 & 29,4 & 30,1 &, 8 & 31,5 & 32,1 & 32,8 & 33,5 & 534,2 & 1,71 \\
\hline 1,72 & 5 & 14,2 & 14,9 & 15,5 & 16,2 & 16,9 & 17,6 & 18,3 & 18,9 & 19,6 & 20,3 & 21,0 & 21,6 & 22,3 & 23,0 & 23,7 & 24,3 & 25,0 & 25,7 & 26,4 & 27,0 & 27,7 & 28,4 & 29,1 & 29,7 & 30,4 & 31,1 & 31,8 & 32,4 & 33,1 & 33,8 & 1,72 \\
\hline 1,73 & 3 & 1 & 14,7 & 1 & 16 & 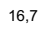 & 4 & 0 & 7 & 19,4 & 0 & 2 & 2 & 1 & 7 & 4 & 24,1 & 24,7 & 25,4 & 26,1 & 26,7 & 27,4 & 28,1 & 28,7 & 29,4 & 30,1 &, 7 & 4 & 2,1 & 32,7 & 733, & 1,73 \\
\hline 4 & 2 & 13,9 & 1 & 15 & 15,9 & 16,5 & 17,2 & 8 &, 5 & 19,2 & 19,8 & 20,5 & 21,1 & 21,8 & 22,5 & 23,1 & 23,8 & 24,4 & 25,1 & 8 & 26,4 & 27,1 & 27,7 & 28,4 & 29,1 & 29,7 & 30,4 & 31,0 & 31,7 & 32,4 & 433,0 & ,74 \\
\hline 175 & 1 & 13,7 & 14,4 & 15,0 & 15,7 & 16,3 & 17,0 & 6 & 3 & 18,9 & 19,6 & 20,2 & 20,9 & 21,6 & 22,2 & 22,9 & 23,5 & 24,2 & 24,8 & 25,5 & 26,1 & 26,8 & 27,4 & 28,1 & 28,7 & 29,4 & 30,0 & 0,7 & 31,3 & 32,0 & 32,7 & 1,75 \\
\hline 1,76 & & 13 & 14 & 14 & 15,5 & 16,1 & 16,8 & 4 & 1 & 18,7 & 19,4 & 20,0 & ,7 & 21,3 & 22,0 & 22,6 & 23,2 & 23,9 & 24,5 & 25,2 & 25,8 & 26,5 & 27,1 & 27,8 & 28,4 & 29,1 & 29,7 & 30,3 & 31,0 & 31,6 & 32,3 & 76 \\
\hline 1,77 & & 13 & 14,0 & 1 & 1 & 1 & 6 & 2 & 17,9 & 18,5 & 19,2 & 19,8 & 20,4 & 21,1 & 2 & 22,3 & 23,0 & 23,6 & 24,3 & 24,9 & 25,5 & 26,2 & 26,8 & 27,5 & 28,1 & 28,7 & 29,4 & 30,0 & 30,6 & 31,3 & $\begin{array}{ll}3 & 31,9\end{array}$ & 1,77 \\
\hline 178 & 6 & 13,3 & 1 & 1 & 1 & 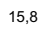 & 4 & 0 & .7 & 3 & 9 & 1 & 20,2 & 20,8 & 21,5 & 1 & 22,7 & 23,4 & 24,0 & 24,6 & 25,2 & 25,9 & 26,5 & 27,1 & 27,8 & 28,4 & 9,0 & 29,7 & 0,3 & 30,9 & 1,6 & 78 \\
\hline 1,79 & 5 & 13,1 & 13,7 & 14,4 & 15,0 & 15,6 & 16,2 & 9 & 7,5 & 18,1 & 18,7 & 19,4 & 20,0 & 20,6 & 21,2 & 21,8 & 22,5 & 23,1 & 23,7 & 24,3 & 25,0 & 25,6 & 26,2 & 26,8 & 27,5 & 28,1 & 28,7 & 29,3 & 30,0 & 30,6 & 31,2 & 1,79 \\
\hline 1,8 & 2,3 & 0 & & & 14,8 & 15,4 & 16,0 & .7 & 3 & 17,9 & 18,5 & 19,1 & 19,8 & 20,4 & 21,0 & 21,6 & 22,2 & 22,8 & 23,5 & 24,1 & 24,7 & 25,3 & 25,9 & 26,5 & 27,2 & 27,8 & 28,4 & 29,0 & 29,6 & 30,2 & $2 \quad 30,9$ & 1,8 \\
\hline & & & & & & & & & & & & & & & & & & & & & & & & & & & & & & & & \\
\hline
\end{tabular}


Tabla II A

Tabla de cálculo del índice ponderal neonatal

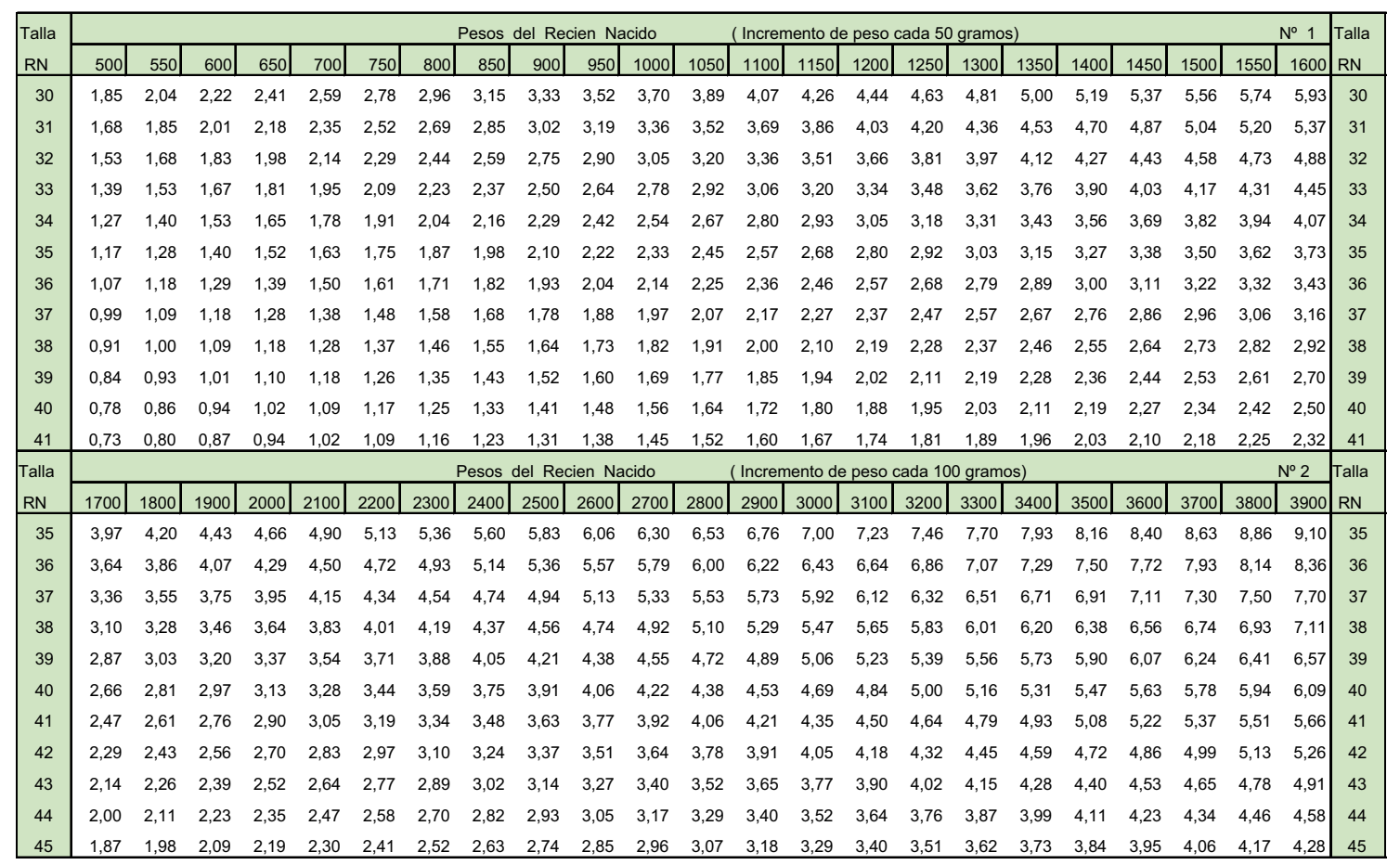

Tabla II B

Tabla de cálculo del índice ponderal neonatal

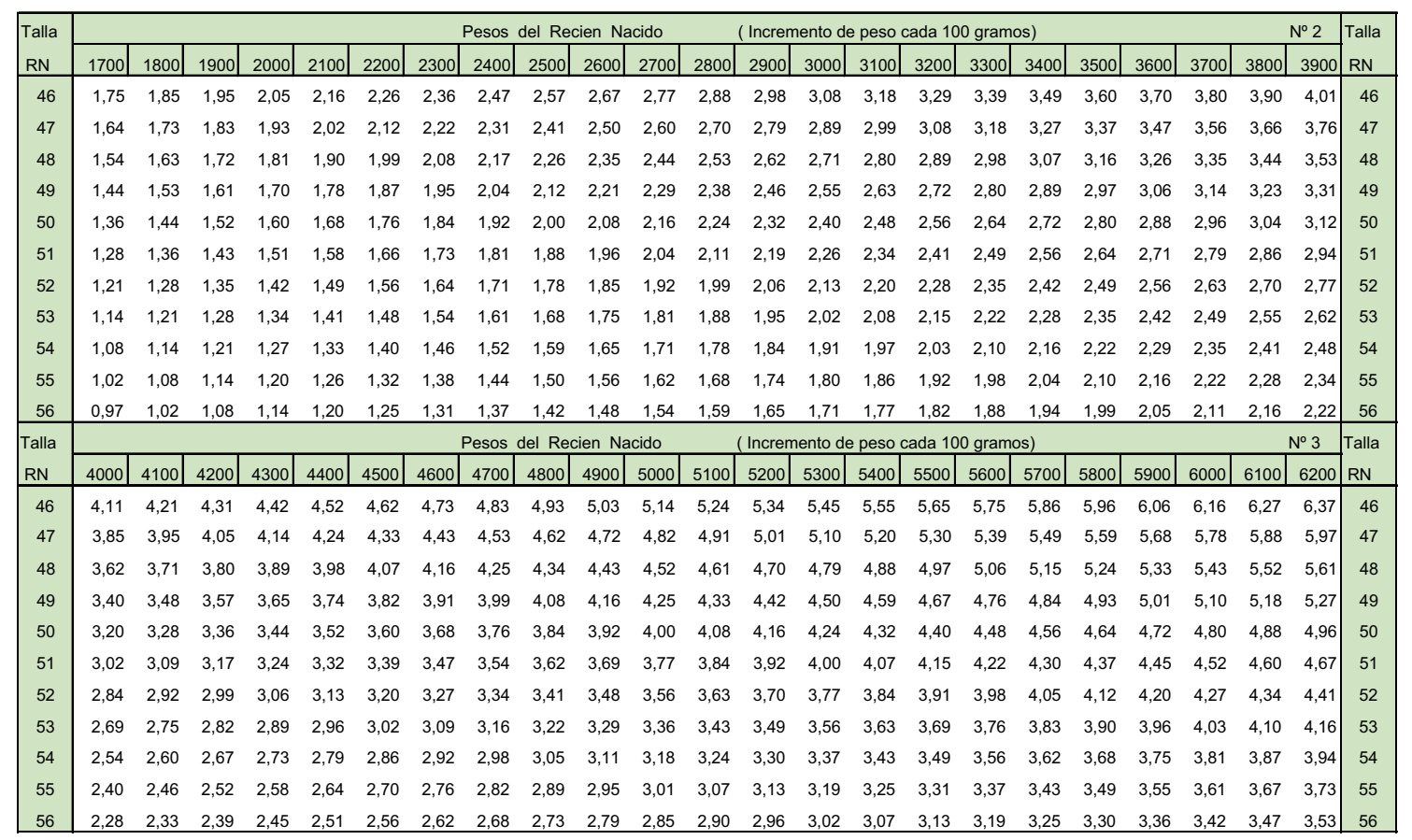




\section{BIBLIOGRAFÍA}

1. Bolzan A, Guimarey L, Norry M. Factores de riesgo de retardo de crecimiento intrauterino y prematurez en dos municipios de Buenos Aires (Argentina). Rev Chil Pediatr 2000;71:255-62.

2. Battaglia FC, Lubchenco LO. A practical classification of newborn infants by weight and gestational age. $\mathrm{J}$ Pediatr 1967;71:159-63.

3. Atalah E, Castro R. Obesidad materna y riesgo reproductivo Rev Méd Chile 2004;132:923-30.

4. Juez G. Curva de crecimiento intrauterino para el diagnóstico apropiado del retardo de crecimiento intrauterino. Rev Med Chil 1989;117:1311.

5. Donoso E, Espinoza R. Efecto de algunas variables nutricionales maternas sobre el peso del recién nacido de término. Rev Latin Perinatol 1988;8:90-4.

6. Abrams B, Parker JD. Maternal weight gain in women with good pregnancy outcome. Obstet Gynecol 1990;76:1-7.

7. Lagos R, Espinoza R, Orellana JJ. Estado nutritivo materno inicial y peso promedio de sus recién nacidos a término. Rev Chil Nutr 2004;31:52-7.

8. Albala C, Vio F, Kain J. Obesidad: un desafío pendiente en Chile. Rev Med Chile 1998;126:1001-9.

9. Mardones F, Rosso P. Desarrollo de una curva patrón de incremento ponderal para embarazadas. Rev Med Chile 1997;125:143-48.

10. Institute of Medicine, National Academy of Sciences. Nutrition during pregnancy. Washington, DC: National Academy Press, 1990.

11. Mardones F, Rosso P, Marshall G, Villarroel L, Bastías $\mathrm{G}$. Comparación de dos indicadores de la relación peso/talla en la embarazada. Acta Pediátrica Española 1999;57:573-8.

12. Atalah E, Castillo C, Castro R. Propuesta de un nuevo estándar de evaluación nutricional en embarazadas. Rev Med Chile 1997;125:1429-36.

13. Campbell S, Thoms A. Ultrasound measurement of the fetal head to abdomen circunference ratio in the assessment of the growth retardation. Br J Obstet Gynaecol 1977;84:165-74.

14. Hadlock FP, Deter RL, Harrist RB, Roecker E, Park
SK. A date-independent predictor of intrauterine growth retardation: femur length/abdominal circumference ratio. AJR 1983;141:979-84.

15. Yagel S, Zacut D, Igelstein S, Palti Z, Hurwitz A, Ro $\neg$ senn B. In utero ponderal index as a prognostic factor in the evaluation of intrauterine growth retardation. Am J Obstet Gynecol 1987;157: 415-9.

16. Juez G, Lucero E, Ventura-Jucá P, Tapia JL, González $\mathrm{H}$, Winter $\mathrm{A}$. Crecimiento intrauterino en recién nacidos chilenos de la clase media. Rev Chil Pediatr 1989;60:198-202.

17. Pittaluga E, Díaz V, Mena $P$, Corvalán $S$. Curva de crecimiento intrauterino para prematuros entre 23 a 36 semanas de edad gestacional. Rev. Chil Pediatr 2002;73:135-41.

18. González R, Gomez R, Castro R, Kae J, Merino P, Etchegaray A, et al. Curva nacional de distribución de peso al nacer según edad gestacional. Chile, 1993 a 2000. Rev Méd Chile 2004;132:115-65.

19. Alarcón J, Alarcón Y, Hering E, Buccioni R. Curvas antropométricas de recién nacidos chilenos Rev Chil Pediatr 2008;79:364-72.

20. Lagos RA, Orellana JJ, Bustos L. Evaluación neonatal del crecimiento intrauterino. Rev Chil Obstet Ginecol 2009;74:209-16.

21. Milad A, Novoa J, Fabres J, Samamé M, Aspillaga C. Recomendación sobre curvas de crecimiento intrauterino Rev Chil Pediatr 2010;81:264-74.

22. Georgieff MK, Sasanow SR. Nutritional assessment of the neonate. Clin Perinatol 1986;13:73-89.

23. Juez G, Lucero E, Ventura-Jucá P. Talla, circunferencia craneana e índice ponderal neonatal en recién nacidos chilenos de clase media. Rev Chil Pediatr 1993;64:237-40.

24. Dombrowski MP, Berry SM, Johnson MP, Saleh AA, Sokol RJ. Birth weight-length ratios, ponderal indexes, placental weights, and birth weight-placenta ratios in a large population. Arch Pediatr Adolesc Med 1994;148:508-12.

25. Caiza Sánchez MT, Díaz Rosselló JL, Simini F. Ponderal index to describe a term neonatal population. An Pediatr (Barc) 2003;59: 48-53. 